\title{
The beauty of resolution: The SN Ib factory NGC 2770 spatially resolved
}

\author{
C. C. Thöne ${ }^{1}$, L. Christensen ${ }^{2}$, J. Gorosabel ${ }^{1,3,4}$ and \\ A. de Ugarte Postigo ${ }^{1,2}$ \\ ${ }^{1}$ Instituto de Astrofísica de Andalucía, Glorieta de la Astronomía s/n, \\ 18008 Granada, Spain. email: cthoene@iaa.es \\ ${ }^{2}$ Dark Cosmology Centre, Juliane Maries Vej 30, DK-2100 Copenhagen, Denmark \\ ${ }^{3}$ Unidad Asociada Grupo Ciencias Planetarias UPV/EHU-IAA/CSIC, Departamento de Física \\ Aplicada I, E.T.S., Ingeniería, Universidad del País Vasco UPV/EHU, E-48013 Bilbao, Spain \\ ${ }^{4}$ Ikerbasque, Basque Foundation for Science, E-48008 Bilbao, Spain
}

\begin{abstract}
The late-type spiral NGC 2770 hosted 3 Type Ib supernovae (SNe) in or next to starforming regions in its outer spiral arms. We study the properties of the SN sites and the galaxy at different spatial resolutions to infer propeties of the SN progenitors and the SF history of the galaxy. Several 3D techniques are used and, for the first time, we present images of metallicity, shocks and stellar population ages from OSIRIS/GTC imaging with tunable narrowband filters.
\end{abstract}

Keywords. supernovae: individual: SN 2008D, SN 2007uy, SN 1999eh; galaxies: abundances; techniques: high angular resolution

\section{Introduction and Observations}

NGC 2770 is a late-type spiral (.SAS5*) at DL $30 \mathrm{Mpc}$ with $\log \mathrm{M}^{*}=10.3 \mathrm{M}_{\odot}$ and $\mathrm{SFR}=1.1 \mathrm{M}_{\odot} / \mathrm{yr}$, largely neglected until the serendipitous discovery of an X-ray outburst (XRO 080109) marking the shock-breakout of SN 2008D (Soderberg et al. 2008). The galaxy had hosted 2 other Type Ib SNe in the past, SN 2007uy and SN 1999eh. Our goal is to infer properties of the SN progenitors by studying their environments and investigate the SF history of the galaxy.

A first study used longslit spectra at 4 slit positions including the SN sites and the center of the galaxy (Thöne et al. 2009). We refine this study using the VIMOS IFU with 0.66 " sampling $(\sim 100 \mathrm{pc})$ at 4 pointings covering $\mathrm{H} \beta$ to $[\mathrm{SII}]$ and tunable narrowband filters at OSIRIS/GTC with 0.25 " sampling ( $\sim 30 \mathrm{pc})$ imaging $[\mathrm{NII}], \mathrm{H} \alpha$ in steps of $12 \AA$ allowing to disentangle the two lines and [SII] in steps of $20 \AA$.

\section{Resolved gas properties}

TF imaging in $\mathrm{H} \alpha$ reveals that NGC 2770 has to be reclassified as a barred spiral galaxy and the bar is also obvious in IR imaging from UKIDSS or 2MASS. Otherwise, the galaxy shows a regular velocity field with some small disturbances or a warp in the SE part of the disk. Some turbulence occurs inside larger SF regions and the ones building the bar.

NGC 2770 shows a smooth negative metallicity gradient. The high resolution TF data reveal gradients within the SF regions whose outskirts are more metal poor. Some projection effects might play a role if the SF regions are not transparent. Shocked material traced by $[\mathrm{SII}] / \mathrm{H} \alpha$ is visible at the edge of SF regions, especially evident in the TF imaging due to the higher resolution. The bar $\mathrm{SF}$ regions have rather low $[\mathrm{SII}] / \mathrm{H} \alpha$ values.

Comparing $\mathrm{H} \alpha$ and an offband filter we can infer the $\mathrm{H} \alpha \mathrm{EW}$ from the TF data, which is related to the age of the SF regions. As expected for inside-out SF there is an age 

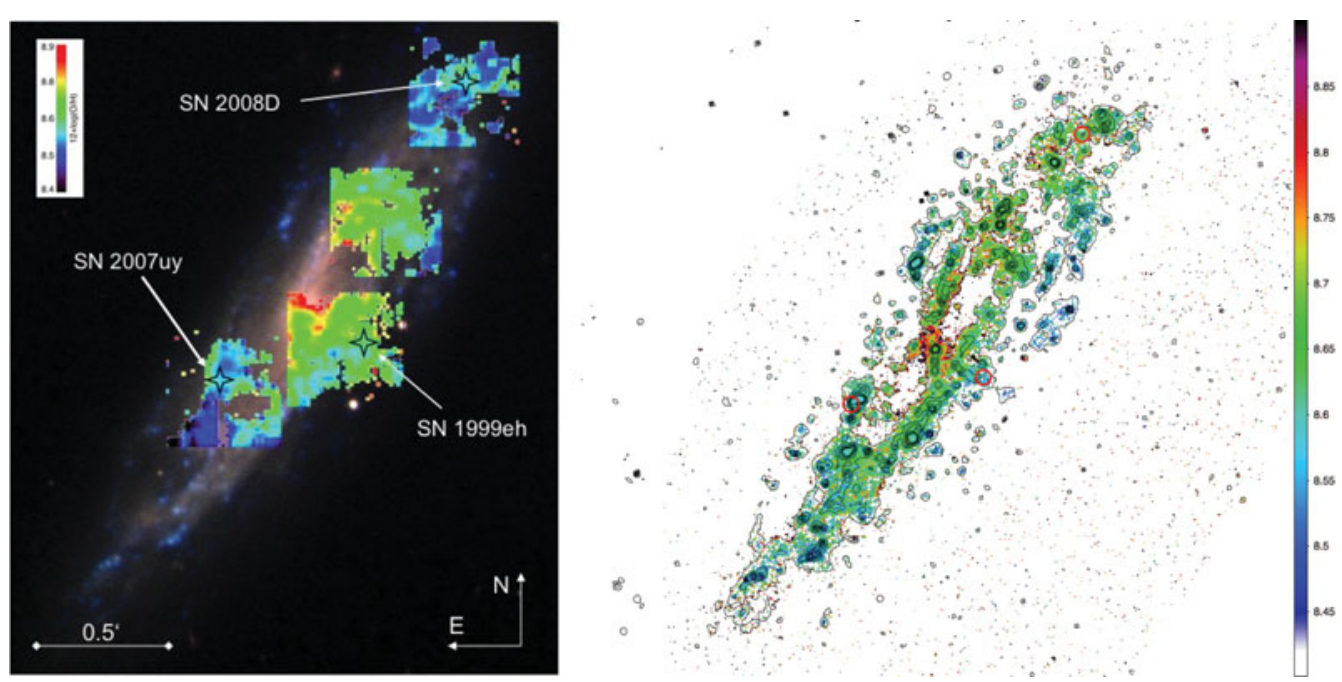

Figure 1. Gas metallicity using the N2 parameter (Marino et al. 2013) in NGC 2770 at different resolutions: Left: VIMOS/IFU, Right: Narrowband TF imaging. The TFs show a large amount of details even within single SF regions averaged out in the lower resolution VIMOS data.

gradient with younger ages in the outskirts. Infact, the youngest regions are found in the third and outermost spiral arm that only stretches across the northern half of the galaxy.

\section{Conclusions on the SN progenitors}

$\mathrm{SNe} \mathrm{Ib}$ are suggested to come from either single WR stars of $\mathrm{M}>35 \mathrm{M}_{\odot}$, corresponding to a lifetime of $\sim 6 \mathrm{Myr}$, or binary systems where the companion strips the star of its $\mathrm{H}$ envelope. Possible differences in metallicity to other SN types are still debated (see e.g. Leloudas et al. 2011; Modjaz et al. 2011). A binary origin is suggested by a recent IFU study (Kuncarayakti et al. 2013), infact, a possible binary companion to a Ib SN has recently been detected in HST imaging (Folatelli et al. 2014; Bersten et al. 2014).

The SN sites in NGC 2770 have $\mathrm{H} \alpha$ EWs of $30-100 \AA$ implying single star progenitors of $<25 \mathrm{M}_{\odot}$. SN 2008D lies on a bridge connecting two SF regions while SN 2007uy and $1999 \mathrm{eh}$ are associated with SF regions, although not the brightest ones. SN 2007uy and 1999eh have metallicities of $12+\log (\mathrm{O} / \mathrm{H}) \sim 8.5$, close to the expected value at their distance from the center, $2008 \mathrm{D}(12+\log (\mathrm{O} / \mathrm{H}) \sim 8.6)$ lies in the upper end of the metallicity at its distance. We conclude that a binary system seems to be a likely progenitor for SNe 2008D and 2007uy while SN 1999eh could be consistent with a single star origin.

\section{References}

Soderberg A. M., et al. 2008, Nature 453, 469

Thöne, C. C., et al. 2009, ApJ, 698, 1307

Marino, R. A., et al. 2013, A\&SA, 559, A114

Leloudas, G., et al. 2011, A\&A,, 530, A95

Modjaz, M., et al. 2011, ApJ, 731, L4

Kuncarayakti et al. 2013, AJ, 146, id. 30

Folatelli, G., et al. 2014, ATEL, 6375

Bersten, M., et al. 2014, astro-ph/1403.7288 